\title{
CFD and experimental studies on the impact of impeller type on flow field and floc size evolution in a stirred tank
}

\author{
Ren Pengfei ${ }^{\mathrm{a}}$ and Nan Jun ${ }^{\mathrm{b}^{*}}$ \\ Skate Key Laboratory of Urban Water Resource and Environment/School of Municipal and \\ Environmental Harbin Institute of Technology, HIT \\ Harbin 150090, PR China \\ apengfeirern84@163.com, bnanjunhiter@163.com
}

\begin{abstract}
Keywords: Computational Fluid Dynamics (CFD), impeller type, velocity gradient (G), floc, Abstract. The impact of impeller type on floc size evolution during flocculation of polystyrene particles with aluminum sulfate has been investigated by both Computational Fluid Dynamics (CFD) and experimental methods. The Lightning R100, A200 and A310 impellers were respectively examined. The results show the impeller type greatly affects the hydrodynamic environment and floc growth process at a given spatially averaged velocity gradient, G. The R100 impeller, as a radial one, can generate great fluid shear force and thus effectively improve floc structure to eventually form large flocs. In contrast, the A200 and A310 impellers, as axial ones, can create great axial-flow circulation that effectively speeds up floc growth at the early stage of flocculation but would not be conductive to the broken-floc restructuring and result in forming small flocs at the later stage. Therefore, the two types of impellers shall be used as a supplement to each other to improve the quality of final flocs. Moreover, the results have proven that the spatially averaged velocity gradient, $\mathrm{G}$, cannot be the single parameter sufficient to model flow field and flocculation efficiency.
\end{abstract}

\section{Introduction}

Flocculation is very widely used as an important process in many industries, including papermaking, mineral processing, and water and wastewater treatment among others [1] [2]. During this process, the hydrodynamic characteristics of particles are altered by turning a large number of small particles into a smaller number of larger ones [3] [4]. Particle aggregation depends on collisions, and the reactor should provide appropriate hydrodynamic conditions to stimulate inter-particle collisions. As flocs grow, they become susceptible to break up by fluid shear. After a certain time, the relationship between floc aggregation and breakage reaches a steady state [5]. Apparently, the flocculation process and the quality of flocs are greatly related to flow field in flocculating reactor.

For the design of coagulation-flocculation reactors, the spatially averaged velocity gradient, G, is generally used to characterize the flow field [6], written as

$$
G=\left(\frac{\varepsilon}{v}\right)^{1 / 2},
$$

where $v$ is the kinematic viscosity of the suspending fluid (here, water), and $\varepsilon$ is the average turbulent energy dissipation rate, described as follows

$$
\varepsilon=\frac{N_{p} n^{3} d^{5}}{V},
$$

where $\mathrm{N}_{\mathrm{p}}$ is the impeller power number, $\mathrm{n}$ is the impeller speed, $\mathrm{V}$ is the liquid volume, and $\mathrm{d}$ is the impeller diameter. Yukselen [7] investigated the effect of shear on the formation and break-up of flocs using conventional jar test procedure and continuous optical monitoring. They found the floc size tended to a nearly constant value after an initial abrupt fragmentation, with only a very slow size reduction over several minutes. Spicer [6] showed that the steady-state average floc size de-pended on the frequency of recirculation to the impeller zone and its characteristic velocity gradient, $\mathrm{G}$. 
Clearly, the flow conditions are heterogeneous, thus the averaged velocity gradient, G, in Eq. (1) does not characterize the local velocity gradients or the variation in flow field in a stirred tank [4]. This is why the use of $\mathrm{G}$ as a design and operating parameter cannot effectively control flocculation efficiency during flocculation process. Fortunately, today Computational Fluid Dynamics (CFD) provides a useful tool for determining detailed information on flow field in a stirred tank. With CFD simulations the local and instantaneous values of flow field can be determined [8] [9] [10]. Visualization of fluid velocity vectors, turbulent kinetic energy and turbulent dissipation rate can greatly help us understand flow field in a stirred tank directly, and identify possible problems in advance.

For a mechanically stirred system, mechanical energy is transferred into the kinetic energy of fluid flow by rotating impeller, generating a certain flow pattern. Therefore, flocculation efficiency is directly related to flow pattern created in the system. However, from literature little study has been done to use the CFD methods to analyze and explore the impact of impeller type on floc growth process in a stirred tank. In our study, CFD simulation has been carried out to characterize the hydrodynamic flow field created by various impellers under given averaged velocity gradients, and CFD predicted and experimental results have been compared to microscopically describe how various impellers would affect floc size evolution during flocculation in a stirred baffled tank.

\section{MATERIAL AND METHODS}

Characteristics of the stirred tank and impellers. All the experiments were carried out in a cylindrical tank (Fig. 1(a)). The homemade tank was made of organic glass with a diameter $\mathrm{T}=150$ $\mathrm{mm}$ and a liquid height $\mathrm{H}=\mathrm{T}=150 \mathrm{~mm}$. Four equally spaced baffles made of glass (width $15 \mathrm{~mm}=$ T/10) were fitted along the internal surface of the vessel. The volume (V) of liquid in the tank was $2.65 \mathrm{~L}$.

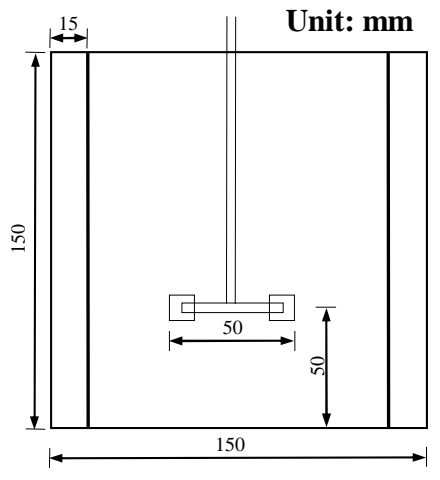

(a) Stirred tank

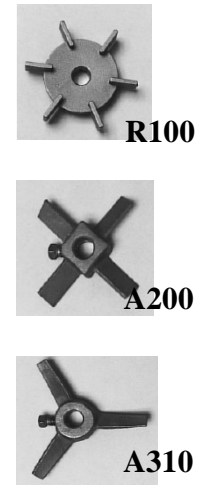

(b) Impellers

Figure 1. Schematic diagram of the stirred tank (a) and photographs of the three impellers (b) used.

The suspension was mixed using one radial flow and two axial flow impellers (Fig. 1 (b)) widely used during flocculation. The radial flow impeller was a high shear radial flow Lightnin R100 impeller, and the two axial flow impellers were a four-blade $45^{\circ}$ pitch Lightnin A200 and a three-blade fluid foil Lightnin A310 impeller. Each impeller had a diameter (d) of $50 \mathrm{~mm}$ and its center was located $50 \mathrm{~mm}$ above the bottom of the tank.

Characterization of flow field in the stirred tank. As the G in Eq. 1 can provide a basis for comparison of the flocculation performance of the employed impellers, it is still used to characterize the macroscopic flow field in this study. The $\mathrm{N}_{\mathrm{p}}$ in Eq. 2 for the three impellers employed is showed in Table 1[6]. In all experiments, the $\mathrm{G}$ was set as 15,25 , and $50 \mathrm{~s}^{-1}$, respectively, during flocculation process, in which case the Reynolds number for each impeller type was larger than 103 so that $\mathrm{N}_{\mathrm{p}}$ value for each impeller is relatively constant. 
Table 1. The impeller power number $\mathrm{Nr}$ for each impeller used.

\begin{tabular}{crrr}
\hline Impeller & R100 & A200 & A310 \\
\hline $\mathrm{Np}$ & 5 & 1.27 & 0.3 \\
\hline
\end{tabular}

Computational strategy. The Cartesian coordinate system was used and the Origin was located at the center of the bottom surface of the tank. The whole tank was selected as computational domain and modeled in GAMBIT to discretize the domain into an unstructured array of tetrahedral mesh elements. Elements were concentrated in the impeller zone to preserve details of velocity shear and increased turbulence.

The standard $\mathrm{k}-\varepsilon$ turbulent model, commonly used in engineering CFD simulations of stirred tanks, was used for all calculations with standard wall function approximations near walls; hence, additional transport equations for turbulent kinetic energy $(\mathrm{k})$ and turbulent dissipation rate $(\varepsilon)$ were solved for these quantities. The standard $\mathrm{k}-\varepsilon$ turbulent model has been successfully used for similar mixing problems by many researchers [8] [9] [11]. Based on the micro-vortex theory, flocculation efficiency is determined by how much energy can be turned into effective energy at each flocculation stage rather than how much energy the main flow can possess in the tank [5]. Thus, the $\mathrm{k}$ and $\varepsilon$ were used to indicate the local turbulence in the flow field and the impact of the flow field on the flocculation efficiency. The k refers to kinetic energy per unit mass, a measurement of the intensity of speed fluctuation. The $\varepsilon$ is the dissipation rate of kinetic energy per unit mass, which is used to indicate the speed when small scale vortices in homogeneous isotropic turbulence transfer mechanical energy into thermal energy. Besides, the impeller rotation were modelled using the Multiple Reference Frame (MRF) impeller model.

A 3-dimension, implicit, segregated, unsteady solver algorithm was adopted to quantify the velocity and turbulence fields. The discretized equations were solved iteratively using the simple algorithm for pressure-velocity coupling, and all terms were discretized by the second-order upwind scheme. The solution was considered converged when the total residuals for the continuity equation dropped to below $10^{-4}$.

Flocculation tests. Flocculation tests conducted by [6] were used to study the impact of impeller type on floc size. In their experiments, an aqueous suspension of monodisperse, spherical, polystyrene particles $\left(\mathrm{d}_{0}=0.87 \mu \mathrm{m}\right)$ was prepared as testing samples. The solids volume fraction was $\Phi=1.4 \times 10^{-5}$, corresponding to an initial particle number concentration of $4 \times 107 \mathrm{~cm}^{-3}$. An acidic stock solution of $0.5 \mathrm{~g} / \mathrm{L}$ of aluminum sulfate hydrate $\left(\mathrm{Al}_{2}\left(\mathrm{SO}_{4}\right)_{3} \cdot 16 \mathrm{H}_{2} \mathrm{O}\right.$; Aldrich, $\left.98 \%\right)$ was selected as the coagulant. All their experiments were conducted using a constant $\mathrm{Al}_{2}\left(\mathrm{SO}_{4}\right)_{3} \cdot 16 \mathrm{H}_{2} \mathrm{O}$ concentration of $10 \mathrm{mg} / \mathrm{L}$, meaning that $76 \%$ of the solids mass present ( $88 \%$ of the solids volume) was contributed by the polystyrene particles and $24 \%$ by the precipitated $\mathrm{Al}(\mathrm{OH}) 3$. Sodium hydrogen carbonate $\left(\mathrm{NaHCO}_{3}\right.$; Aldrich, 99\%) was used to buffer the suspension and the $\mathrm{pH}$ was kept at $7.2 \pm 0.05$ during all experiments. The polystyrene suspension was first mixed at $G=300 \mathrm{~s}^{-1}$ for five minutes to break up any agglomerates. The coagulant was then added and mixed with the suspension for one minute. The impeller was then set to the desired speed, corresponding to $G=15,25$ and $50 \mathrm{~s}^{-1}$, respectively. Samples were removed for size analysis using the wide end of a $0.5-\mathrm{cm}$ ID pipette and floc size distributions were measured by image analysis.

\section{RESULTS AND DISCUSSION}

First it is important to investigate the flow pat-terns created by the three impellers as the impeller type greatly affects the hydrodynamic environment in a stirred tank. Figure 2 shows velocity vectors of axial cross-section created by the R100, A200 and A310 impellers, respectively, in the stirred tank at $\mathrm{G}=15 \mathrm{~s}^{-1}$. The flow field created by the R100 impeller in the stirred tank shows the typical 
characteristics of "double circulation" flow configuration, i.e., the high radial turbulent generated by the impeller splits into two parts after hitting the wall of the stirred tank, with one moving upward along the wall and the other downward, creating two circular flows in the tank (Fig. 2(a)). The flow created by the A310 impeller is rapidly "spinned off" in the vicinity of the impeller and squeezed and pressed by the impeller with greater axial component. The flow field in the stirred tank has shown the characteristics of an axial flow, i.e., the "integrated circulation" flow configuration; especially at the bottom of the tank where the circular flows at the near-wall wash-out zone have stronger circulating turbulent that can effectively drive the sedimentary flocs to the impeller zone with higher mixing intensity (Fig. 2(c)). As seen in Figure 2(b), the A200 impeller produces flow that is a combination of axial and radial patterns.

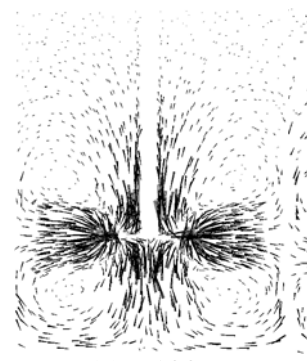

(a) R100

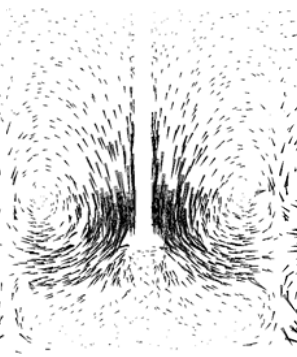

(b)A200

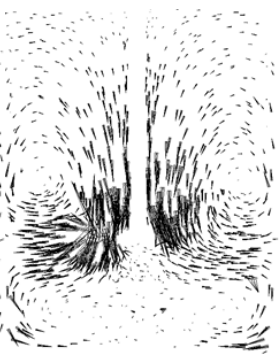

(c)A310

Figure 2. Velocity vectors of axial cross-section created by the employed impellers in the stirred tanks at $\mathrm{G}=15 \mathrm{~s}^{-1}$ : (a) R100, (b) A200, (c) A310.

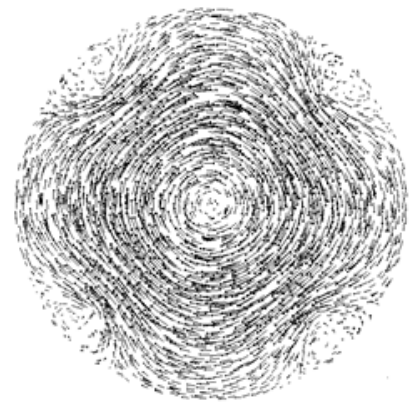

(a) $Z=0.250 H$

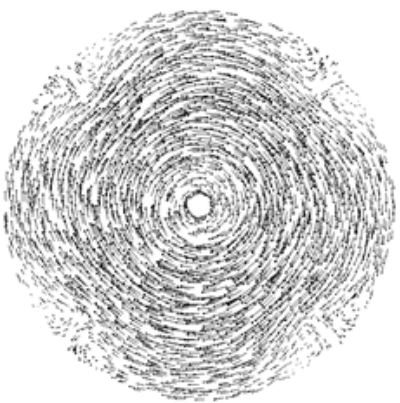

(b) $Z=0.500 H$

Figure 3. Velocity vectors of horizon cross-sections of (a) $\mathrm{Z}=0.125 \mathrm{H}$ and (b) $\mathrm{Z}=0.500 \mathrm{H}$ created by the Lightnin R100 impeller in the stirred tank at $\mathrm{G}=15 \mathrm{~s}^{-1}$.

Figure 3 shows velocity vectors of horizon cross-sections of $\mathrm{Z}=0.250 \mathrm{H}$ and $0.500 \mathrm{H}$ created by the R100 impeller in the stirred tank at $\mathrm{G}=15 \mathrm{~s}^{-1}$. It can be seen that due to the baffle disturbance, the flow becomes unordered in its vicinity and creates small swirls behind the baffle, preventing the flow from creating an integrated "swirl" in the stirred tank. It also weakens the shearing flow that adversely affects the mixing result and strengthens the radial and axial flows that are helpful for mixture. Therefore, it is necessary to install baffles for stirring systems of a certain size.

As shown in the discussion on the flow patterns, different impeller types create different flow patterns, and thus the turbulent velocity and kinetic energy fields differ remarkably. Figure 4 and Figure 5 show contours of velocity magnitude and turbulent kinetic energy of axial cross-section for all three impellers at $\mathrm{G}=15 \mathrm{~s}^{-1}$. 


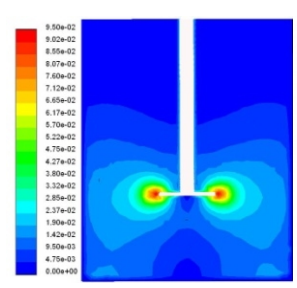

(a) R100

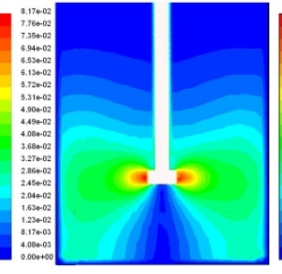

(b)A200

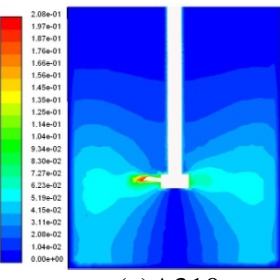

(c)A310

Figure 4. Contours of velocity magnitude of axial cross-section created by the employed impellers in the stirred tanks at $G=15 \mathrm{~s}^{-1}$ : (a) R100, (b) A200, (c) A310. Each color on the spectrum on the left side corresponded to the value of velocity.

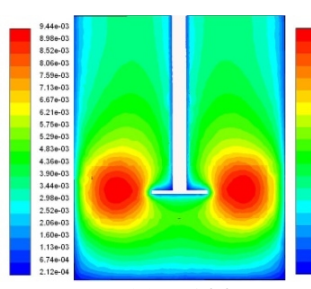

(a) R100

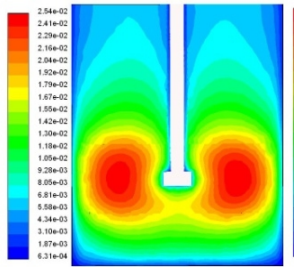

(b)A200

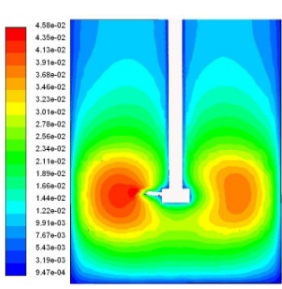

(c)A310

Figure 5. Contours of turbulent kinetic energy of axial cross-section created by the employed impellers in the stirred tanks at $G=15 \mathrm{~s}^{-1}$ : (a) R100, (b) A200, (c) A310. Each color on the spectrum on the left side corresponded to the value of kinetic energy.

Figure 6 and Figure 7 show the distributions of average turbulent kinetic energy and turbulent dissipation rate of different distances from the horizon cross-section to the bottom surface of the tank for each impeller at $\mathrm{G}=15,25$ and $50 \mathrm{~s}^{-1}$.

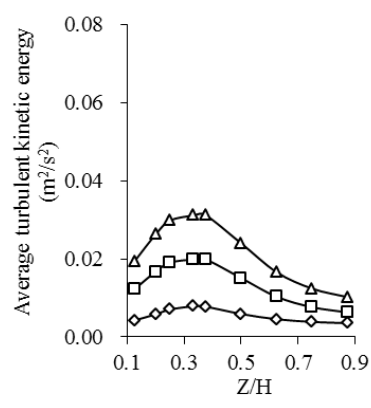

(a) $G=15 \mathrm{~s}^{-1}$

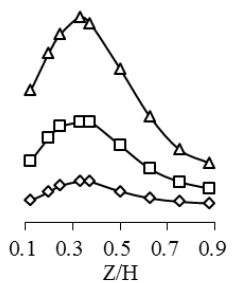

(b) $G=25 \mathrm{~s}^{-1}$

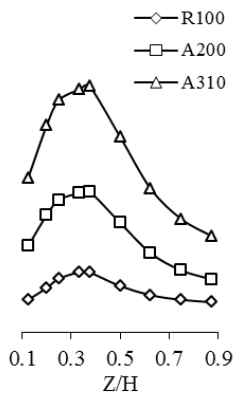

(c) $G=50 \mathrm{~s}^{-1}$

Figure 6. Distributions of average turbulent kinetic energy in the stirred tank for all three impellers at $\mathrm{G}=$ (a) 15 , (b) 25 , and (c) $50 \mathrm{~s}^{-1}$.

For a certain type of impeller, the stirring energy will be transmitted gradually from the impeller zone to other areas, therefore the velocity at the tip of the impeller is the highest, which decreases in the vicinity of the impeller and becomes the lowest in distant areas to the impeller (especially around the free liquid surface) (Fig. 4). Figure 5 and Figure 6 show that the turbulent kinetic energy is the highest in the vicinity of the impeller in the stirred tank and gradually decreases when reaching out from the impeller zone. The turbulent kinetic energy is also high in the near wall area and becomes lowest around the liquid surface and the bottom of the tank, indicating that kinetic energy is basically generated in the vicinity of the impeller where turbulent fluctuation is greater and brings about better mixing results. As shown in Figure 7, for each impeller, the turbulent dissipation rate is also the highest in the vicinity of the impeller which rapidly decreases when reaching out from the area and becomes the lowest in the circulating area beyond the zone, indicating that the turbulent dissipation centers around the impeller zone where the microscopic mixing occurs and heat energy is trans-formed at high frequency. 


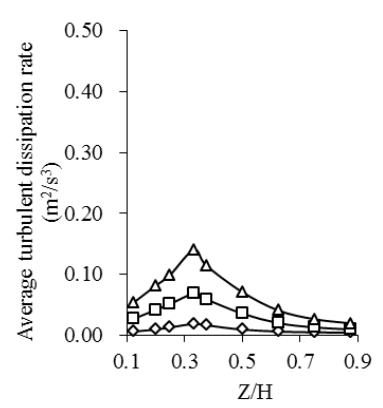

(a) $G=15 \mathrm{~s}^{-1}$

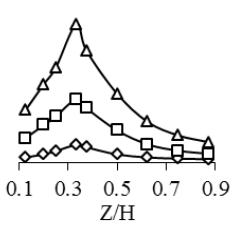

(b) $G=25 \mathrm{~s}^{-1}$

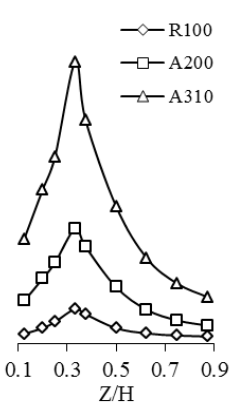

(c) $G=50 \mathrm{~s}^{-1}$

Figure 7. Distributions of average turbulent dissipation rate in the stirred tank for all three impellers at $\mathrm{G}=$ (a) 15 , (b) 25 , and (c) $50 \mathrm{~s}^{-1}$.

Figure 6 and Figure 7 also show, at a constant G, the A310 impeller produces the severest turbulent kinetic effect, followed by the A200 and R100 impellers. Moreover, for a certain type of impeller, as $\mathrm{G}$ increases, the differences in the distributions of average turbulent kinetic energy and turbulent dissipation rate within different distance ranges becomes more pronounced. Average turbulent kinetic energy and turbulent dissipation rate in the impeller zone and in the vicinity of the impeller $(\mathrm{Z} / \mathrm{H}=$ 0.25 0.50) increase noticeably while those in distant zones remained low and changed only moderately

Figure 8 shows experimental results of the floc size evolution at different velocity gradients for all the impellers. At a given G, floc growth, initially, is dominant so the average floc size rapidly increases. As the floc becomes larger, its ability to withstand the shear force becomes weaker and the flocs break apart. When the floc breakage becomes significant and competes with aggregation, the rate of floc growth begins to slow down. Later on, the average floc size levels off because the relationship of floc aggregation and breakage reaches a steady state. Figure 8 also shows that the impeller type affects the floc size evolution considerably. At $G=15 \mathrm{~s}^{-1}$, a steady state floc size is reached first by the A200 impeller, followed by the A310 and R100 impellers. However, the largest flocs are produced by the Lightnin R100 impeller (Fig. 8(a)). Figure 8(b) shows that steady state is attained faster at $\mathrm{G}=25 \mathrm{~s}^{-1}$ than at $\mathrm{G}=15 \mathrm{~s}^{-1}$, and, however, a significant decrease in the steady state floc size occurs with the increase in the velocity gradient. At $G=50 \mathrm{~s}^{-1}$, a sharp decrease in the average floc size appears before entering into the steady state for both the A200 and A310 impellers, but, with the R100 impeller, this phenomenon is not remarkable (Fig. 8(c)).

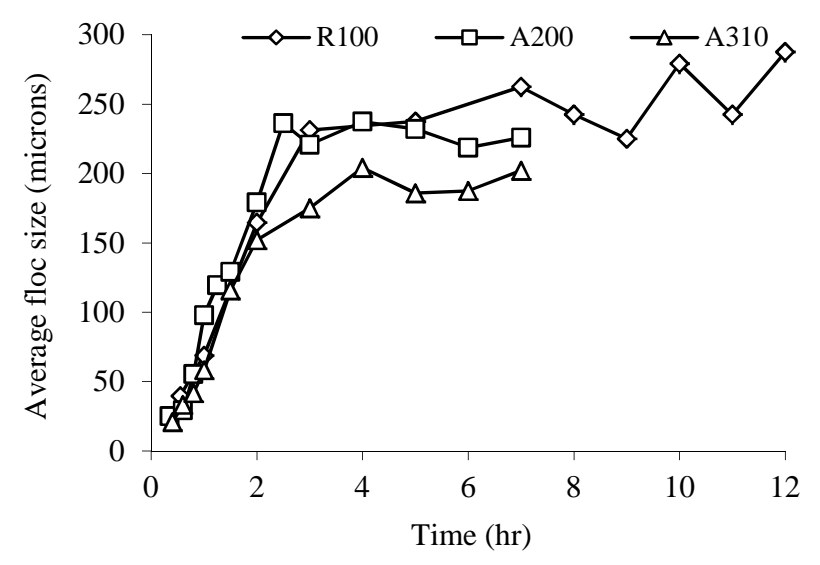

(a) $\mathrm{G}=15 \mathrm{~s}^{-1}$ 


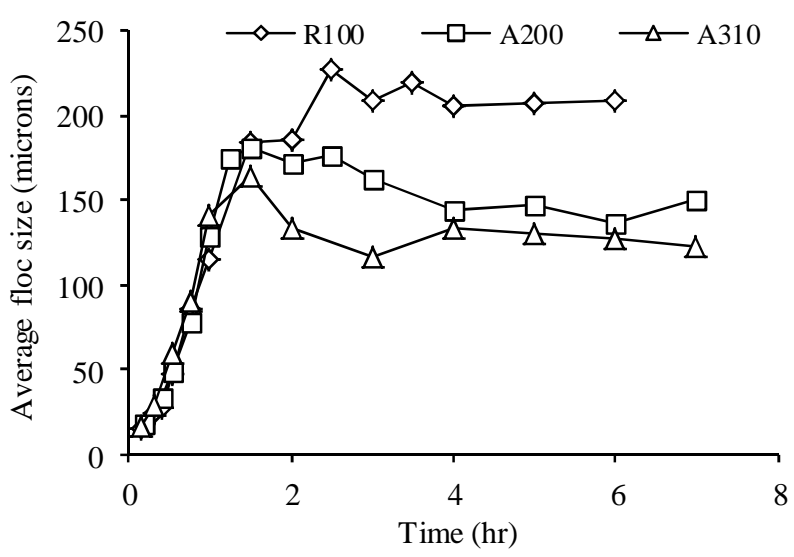

(b) $\mathrm{G}=25 \mathrm{~s}^{-1}$

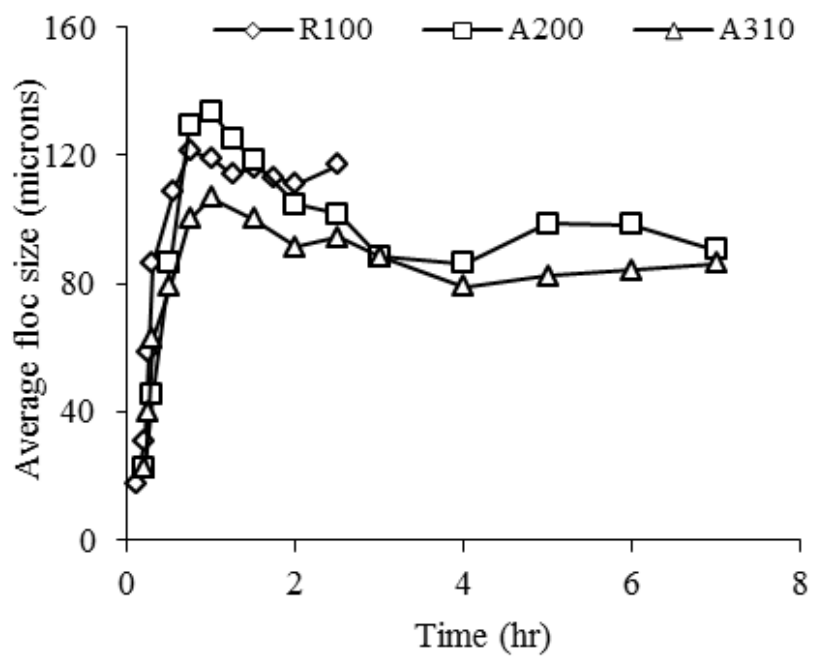

(c) $G=50 \mathrm{~s}^{-1}$

Figure 8. Evolution of floc size in the stirred tank for all three impellers at $G=$ (a) 15, (b) 25, and (c) $50 \mathrm{~s}^{-1}$. The experimental data came from the paper written by Spicer (6)

Most previous studies have treated the floc growth process as the combined processes of floc aggregation, breakage and reformation, shown in Figure 9 [12]. Agglomerating particles must firstly collide with each other and secondly must adhere upon collision. Aggregation takes place at a rate that depends on the collision frequency and the fraction of collisions which result in attachment. However, flocs do not continue growing and further growth is restricted by the given $G$ for essentially two reasons. As particle size increases, the collision efficiency of particles in a shear field becomes lower and floc breakage occurs significantly because of the inherent complexity and fragility of flocs [7]. Of course, some broken fragments can be reformed to some extent. As a result, a steady-state floc size distribution is obtained, where the limiting size is governed by the aggregation, breakage and restructuring processes.

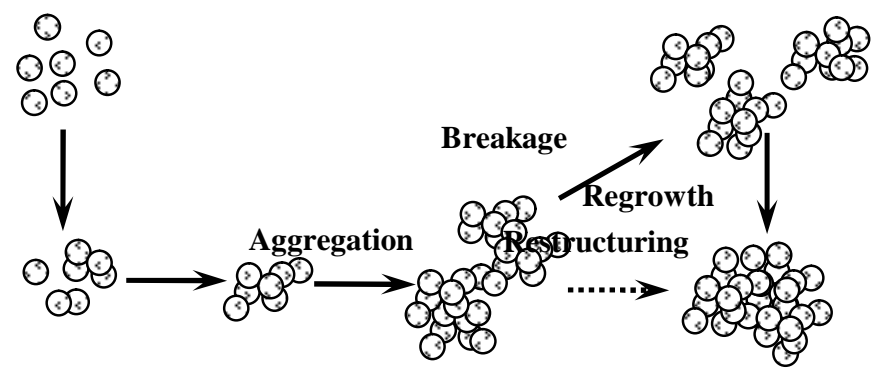

Figure 9. Schematic of floc growth process at a given velocity gradient. 
As discussed above, the impeller type greatly affects the hydrodynamic environment. In the stirred tank, the spatially inhomogeneous distribution of the turbulent fluctuation and the spatial variance of the local turbulent dissipation rate result in the spatially inhomogeneous distribution of the local velocity gradient and eventually the spatially inhomogeneous distribution of the shearing rate. The role of the impeller is mainly to impose the shearing force on the flow and stimulate the flow to circulate in the tank. The axial flow impellers (the A200 and A310 impellers) produce a constant pumping action toward the bottom of the tank followed by circulation to the top and return to the impeller zone, showing strong capabilities for integrated circulating that improves the transmission of stirring energy and the macroscopic mixing of the materials in the stirred tank. In contrast, the radial flow impeller (the R100 impeller) creates fluid flow directed radial out from the impeller, which, once collided onto the wall of the tank, causes the energy to dissipate and the power to lose, showing strong shearing force. Therefore, the impeller should be applied to stirring process that requires higher shearing force. At the early stage of floc growth process, large flocs are beginning to form but small clusters are still widespread [4]. The A200 and A310 impellers can improve the overall circulating results in the stirred tank, and effectively increase the chances for the floc particles to collide and the adhesion between particles so that the flocs forms rapidly. However, while the intense turbulent fluctuation makes the existing flocs break up at the later stage of flocculation, it does not generate the effective restructuring of the particles. The reason may be that the larger flocs are broken into many smaller flocs of similar size, which have identical or similar inertia making them difficult to collide and cohere effectively so that the average floc size is smaller at the later stage. For the R100 impeller, its strong shearing force can improve the effective collision between particles in water, and meanwhile effectively reduce the water content in existing flocs so that dense flocs are formed and can resist the shearing force during the breakage-restructuring process at the later stage of flocculation. Moreover, the strong shearing force can just cause the tiny particles on the surface of the flocs to fall off. Since existing flocs are different in size and differ greatly in inertia, the floc particles show stronger reformation capabilities and eventually form into larger size.

\section{CONCLUSIONS}

In the present work, both CFD and experimental methods have been used to investigate the impact of impeller type on flow field and the polystyrene-alum floc size evolution produced at various spatially averaged velocity gradients, $\mathrm{G}$, of 15,25 and $50 \mathrm{~s}^{-1}$. CFD modeling was carried out to model the flow field created by various impeller types at a given spatially averaged velocity gradient, G. Based on the CFD predicted results, and characteristics of velocity magnitude, turbulent kinetic effect, etc, of flow field created by various impeller types were contrastively analyzed. Then, the results of experiments conducted by Spicer [6] were used to study how different impellers affected the floc size evolution.

The CFD and experimental results show that the impact of radial and axial flow impellers on the floc size evolution differs largely due to their different stirring characteristics. The axial flow impellers (the A200 and A310 impellers) have greater axial circulating power than the radial one especially around the margin of the impeller blade so that they can effectively enhance the velocity of floc growth at the earlier stage of flocculation but are not improving the restructuring of broken flocs at the later stage, resulting in smaller floc size. The radial flow impeller (the R100 impeller) has greater shearing power and can effectively improve the floc structure to form large and dense flocs that can resist the shearing breakage at the later stage of flocculation and form particles into larger flocs. The flocculation process is a complex physicochemical process during which the floc particles keep colliding, growing and being broken apart by shearing force. Therefore, to improve the floc size evolution, it is suggested that the axial flow impellers (the A200 and A310 impellers) shall be employed at the earlier stage of flocculation to expedite the flow growth while the radial flow impeller (the R100 impeller) at the later stage to improve the formation of large and dense flocs. The two types of impellers can be used together as a supplement to each other. 
The CFD and experimental results also show that the flow configuration and flocculation efficiency in the stirred tank is not only related to the spatially averaged velocity gradient, G, but also to the geometrical characteristics of the impeller such as the impeller type. Therefore, it has proven again that the adoption of $\mathrm{G}$ as a design and operating parameter in the experimental or engineering process has a certain limitation

\section{ACKNOWLEDGEMENTS}

This study is supported by State Key Laboratory of Urban Water Resource and Environment (Harbin Institute of Technology) (No 2012DX07)

\section{References}

[1] Yao M, Nan J, Chen T. Effect of particle size distribution on turbidity under various water quality levels during flocculation processes. Desalination. 354 (2014) 116-24.

[2] Vlieghe M, Coufort - Saudejaud C, Frances C, Liné A. In situ characterization of floc morphology by image analysis in a turbulent Taylor-Couette reactor. AIChE Journal. 60 (2014) 2389-403.

[3] Nasser M. Characterization of floc size and effective floc density of industrial papermaking suspensions. Separation and Purification Technology. 122 (2014) 495-505.

[4] Coufort C, Dumas C, Bouyer D, Liné A. Analysis of floc size distributions in a mixing tank. Chemical Engineering and Processing: Process Intensification. 47 (2008) 287-94.

[5] Jarvis P, Jefferson B, Gregory J, Parsons SA. A review of floc strength and breakage. Water Research. 39 (2005) 3121-37.

[6] Spicer PT, Pratsinis SE. Shear-induced flocculation: the evolution of floc structure and the shape of the size distribution at steady state. Water Research. 30 (1996) 1049-56.

[7] Yukselen MA, Gregory J. The reversibility of floc breakage. International Journal of Mineral Processing. 73 (2004) 251-9.

[8] Deglon DA, Meyer CJ. CFD modelling of stirred tanks: Numerical considerations. Minerals Engineering. 19 (2006) 1059-68.

[9] Javed KH, Mahmud T, Zhu JM. Numerical simulation of turbulent batch mixing in a vessel agitated by a Rushton turbine. Chemical Engineering and Processing: Process Intensification. 45 (2006) 99-112.

[10] Becker V, Schlauch E, Behr M, Briesen H. Restructuring of colloidal aggregates in shear flows and limitations of the free-draining approximation. Journal of colloid and interface science.339 (2009) 362-72.

[11] Yu W, Li G, Xu Y, Yang X. Breakage and re-growth of flocs formed by alum and PACl. Powder Technology. 189 (2009) 439-43.

[12] Jarvis P, Jefferson B, Gregory J, Parsons SA. A review of floc strength and breakage. Water Research. 39 (2005) 3121-37. 